\title{
Production of Gliders by Collisions in Rule 110
}

\author{
Genaro Juárez Martínez ${ }^{1}$, Harold V. McIntosh ${ }^{2}$, and \\ Juan Carlos Seck Tuoh Mora ${ }^{3}$ \\ 1 Departamento de Ingeniería Eléctrica, Sección Computación, \\ CINVESTAV-IPN, Av. IPN 2508, San Pedro Zacatenco, \\ Apartado Postal 14-740, 07360, México D.F. \\ genarojm@correo.unam.mx \\ 2 Departamento de Aplicación de Microcomputadoras, \\ Instituto de Ciencias, Universidad Autónoma de Puebla, \\ Apartado Postal 461, 72000, Puebla, Puebla México \\ http://delta.cs.cinvestav.mx/ mcintosh \\ 3 Centro de Investigación Avanzada en Ingeniería Industrial, \\ Universidad Autónoma del Estado de Hidalgo, \\ Carr. Pachuca-Tulancingo Km. 4.5, \\ Pachuca Hidalgo 42184, México \\ jseck@uaeh.reduaeh.mx
}

\begin{abstract}
We investigate the construction of all the periodic structures or "gliders" up to now known in the evolution space of the onedimensional cellular automaton Rule 110. The production of these periodic structures is developed and presented by means of glider collisions. We provide a methodology based on the phases of each glider to establish the necessary conditions for controlling and displaying the collisions of gliders from the initial configuration.
\end{abstract}

\section{Introduction}

The interest in the study of Rule 110 begins with the investigations by Stephen Wolfram in one-dimensional cellular automata. Wolfram detects that this automaton can support complex behaviors identifying the existence of well-defined periodic structures in the evolution space, as Douglas Lind describes in the appendix of [21]. In the cellular automata environment, a periodic structure moving through time is called a glider.

Cellular automata are discrete dynamical systems which evolve through time, these systems may support complex and self-reproducing behaviors, as it is described by the precursor of this theory John von Neumann in [20].

The most famous cellular automaton is The Game of Life developed by John Horton Conway [6], it is a binary two-dimensional automaton and it has been used to implement artificial life, for instance, state zero represents a dead cell and state one a live one. In this context the initial configuration has a number of live beings who vary from generation to generation applying the evolution rule, reproducing a set of very interesting behaviors. 
The wide variety of behaviors presented by this type of automata is the reason to investigate and simulating certain biologic, chemical, mathematics, physics and computing processes 1 . In the computing theory environment, Conway demonstrates that The Game of Life is universal on simulating a registry machine, constructing logics gates with gliders [2].

An important result in cellular automata theory in the last twenty years is developed by Matthew Cook in the middle of the 90's. Cook proves that 110 is universal by means of simulating a cyclic tag system [3, 22] y [12. This demonstration and the one corresponding with The Game of Life use gliders to represent data and operations.

Cook establishes a classification of the gliders in Rule 110, the list was available in 4$]^{2}$ and one part of the same appears in [22]. The list of gliders proposed by Cook is more complete than the one presented by Lind, because it includes quite a rare extensions of gliders, gliders of complex construction and the existence of a glider Gun.

In The Game of Life the glider Gun is very important to construct the registry machine, but in Rule 110 the glider Gun does not have an relevant paper in this sense. In this case blocks of well-defined gliders are used to simulate each one of the parts of the cyclic tag system. This is a direct application of the gliders, although the existence of gliders is an interesting subject by its own sake. For instance, the number of collisions of gliders in Rule 110 is unlimited because gliders can be grouped for traveling together in the evolution space and some of them have extensions, this is important because several complex processes may be implemented [1.

One of the questions is to know if Rule 110 can reproduce by itself each one of its periodic structures, we solve this problem for gliders without extensions.

In this paper we show that each glider (without extensions) proposed by Cook is obtained by collisions, using glider phases aligned by ether. In order to reproduce each collision we use the phases [8] [12] to control gliders by means of establishing a horizontal measurement in the initial configuration. We take the binary productions presented in [10] for classifying all the binary collisions producing a particular glider, this detailed examination demonstrates that $\mathrm{D}_{2}$, $\operatorname{Bbar}_{n}, \mathrm{Bbar}_{n}$ and $\mathrm{H}$ gliders and the glider Gun cannot be obtained through binary collisions, but they are product of multiple collisions. Throughout this paper we use the classification proposed by Cook to identify each glider.

The collisions illustrated in this work were yielded by the OSXLCAU21 system which may be freely obtained in [24]. This system applies the glider phases to construct suitable initial configurations in Rule 110, and it also allows to filter ether using an adequate selection of colors.

\footnotetext{
${ }^{1}$ In the web site of Tim Tyler there are several examples including attractive applets and other directions with excellent works in these subjects, http://timtyler.org/

${ }^{2}$ By some legal problems, the list and some other information developed by Cook about Rule 110 were retired 7 .
} 


\section{Representation of Rule 110 by Means of Phases}

A relevant feature of Rule 110 is that it is a binary one-dimensional cellular automaton with a very simple local dynamics, but the global behaviors that the rule is able to construct are highly complex. In this way, we shall provide the basic terms used in cellular automata theory.

A one-dimensional cellular automaton is composed by a linear array of cells where each cell takes one value from 0 or 1 , this is the initial configuration of the system. The evolution rule is defined by the transformations of the neighborhoods, a neighborhood is formed by a central cell, a neighbor to the right and another to the left. In order to calculate the following configuration the evolution rule is applied to all neighborhood in the array at the same time. This process is repeated a number of times producing the global evolution of the automaton; an example of the global evolution of Rule 110 using a random initial configuration is illustrated in Figure 1 .

The number 110 represents the rule in decimal notation which originally is the binary number 01110110, in this way the neighborhoods 000, 100 and 111 evolve into 0 in the following generation and the neighborhoods $001,010,011$, 101 and 110 evolve into. Notice that the ending cells of the ring at both sides specify incomplete neighborhoods, in order to solve this problem we concatenate the initial cell with last one to have complete neighborhoods in each position of the evolution space.

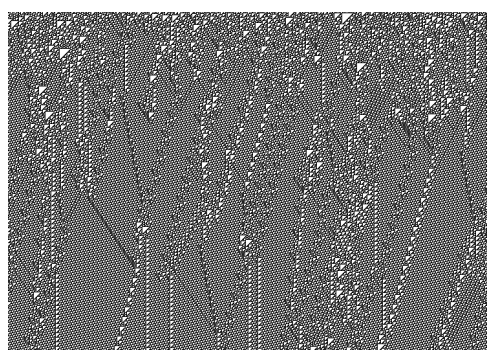

Fig. 1. Random evolution in Rule 110.

In Figure1 we have regions with stable behaviors represented by the periodic background called ether by Cook, the periodic regions are determined by gliders and the chaotic regions may be generated from the initial configuration or as product of collisions with a short or long duration. In this figure we can also see all the possible gliders which arise in a natural way as Lind describes in the appendix of [21].

The evolution of Rule 100 can be seen as a covering of the evolution space by means of triangles formed by the cells of the automaton, this is defined by Harold V. McIntosh in [15]. $\mathrm{T}_{n}$ defines a triangle where very side has $n$ cells for 
$n \in \mathbb{Z}^{+}$; an interesting question is to determine the largest triangle produced by a collision in Rule 110 [16].

Ether is represented by a periodic sequence which moves 14 cells to the right in 7 generations. A triangle $\mathrm{T}_{3}$ represents ether and it establishes our horizontal measurement aligning a pair of them to obtain a periodic sequence. We shall first represent in a systematic way each one of gliders classified by Cook and then we shall control each production using the glider phases. For this reason we shall establish a horizontal measurement $\mathrm{f}_{i-} i$ for $1 \leq i \leq 4$ as Figure 2 illustrates.

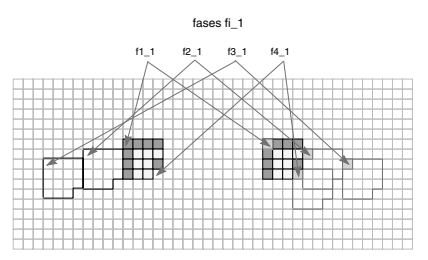

Fig. 2. Phases in Rule 110.

For instance, the sequence 11111000100110 represents the phase $f_{1} \_1$ of ether and we describe it as $\mathrm{e}\left(\mathrm{f}_{1-1} 1\right)$. This alignment allows to identify four different periodic sequences $\mathrm{f}_{i}$ to represent a given particular glider. In the case of gliders with more than one alignment with ether, they have several phases to initiate from the initial configuration. The second subindex $i$ indicates the phase of the $\mathrm{T}_{3}$ triangle; the other phases are a permutation of the first one. Therefore the $\mathrm{f}_{i-1} 1$ phases are enough to establish the horizontal measurement.

Gliders are codified in the following way: $\#_{1}\left(\#_{2}, \mathrm{f}_{i-1} 1\right)$, where $\#_{1}$ represents a glider according to the classification of Cook and $\#_{2}$ is the phase of the glider if it is greater than one.

\section{$3 \quad$ Producing Gliders by Means of Collisions}

Gliders arising in a natural way in the evolution space are not difficult to obtain analyzing all the binary collisions; but Cook mentions that there are gliders which cannot be produced in the evolution of the automaton and they can only be specified from the initial configuration. However, although the Bbar8 and $\mathrm{H}$ gliders and the glider Gun are indeed complicated structures which do not arise commonly in the evolution space, they can be generated by means of collisions. In this sense specialized computing searches were developed, finding several interesting results.

Figure 3 depicts the production of each glider and the lower part of each figure describes the sequence codified in phases to produce each collision. In the case of the Bbar8 glider the collision is complicated and the synchronization of each one of its parts is unique; the change of a single cell disturbs completely 

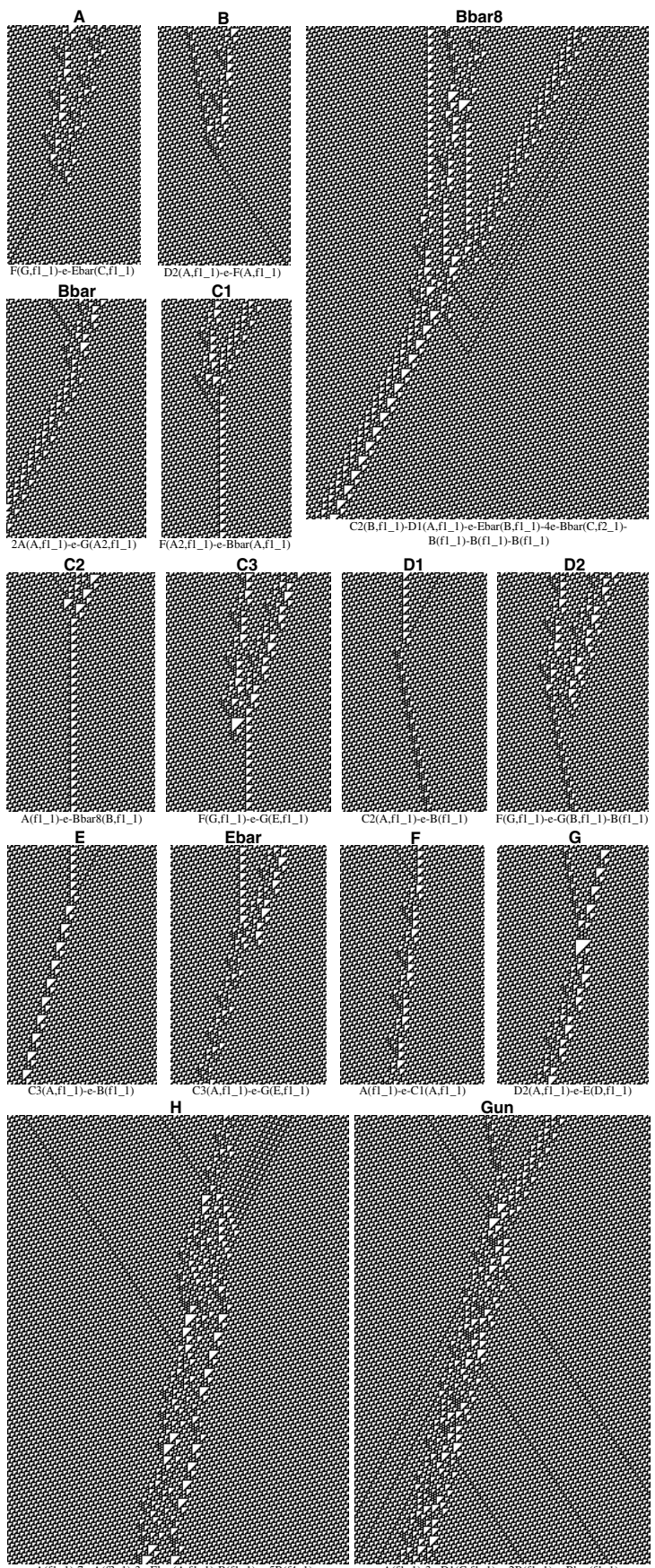

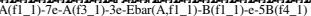

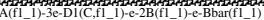

Fig. 3. Gliders of Rule 110 reproduced by collisions. 
the final result. The same phenomenon is presented on synchronizing the gliders to simulate a cyclic tag system 17 .

An important point is that the succession of collisions must be defined taking into account the order of the glider speeds. For instance in order to generate the Bbar8 glider, the $\mathrm{C}_{2}$ and $\mathrm{D}_{1}$ gliders have speed of 0 and of $1 / 5$ respectively. 3 . Thus the existence of the $\mathrm{D}_{1}$ glider preceded by the $\mathrm{C}_{2}$ glider may be questioned whether it is product of a collision or not.

The A glider is generated by the Ebar and F gliders, the most of the collisions between these gliders have a soliton-like behavior. 4 Solitons have their own interest in cellular automata theory as Kenneth Steiglitz describes in 18 and [13]. However it does not imply that they are the only gliders in Rule 110 able to simulate solitons [9].

The $\mathrm{G}$ glider is produced by an isolated triangle $\mathrm{T}_{13}$, an relevant question is to know if each glider may be yielded by an isolated triangle. For instance the $\mathrm{T}_{10}$ triangle induces an Ebar glider, the $\mathrm{T}_{8}$ defines an $\mathrm{E}$ and the $\mathrm{T}_{1}$ generates the $\mathrm{A}$ and $\mathrm{B}$ gliders.

$\mathrm{H}$ glider is produced by internal collisions among several gliders and small chaotic regions interacting at the same time; in this sense we can suppose the existence of more complex gliders. An important restriction is that every glider must advance with increments of $2 / 3$ and going back with decrements of $-1 / 2$ in each phase. On the other hand although we have a complex glider in its construction, it can cover the evolution space and sometimes without ether [11; for instance the $\mathrm{H}$ glider has two ways of covering the evolution space yielding a really exotic result.

Finally, the existence of a glider Gun is important in two aspects: the first is the straightforward representation of the unlimited growth of the automaton, and the second is the possibility of constructing a self-reproducing system, a relevant result developed by von Neumann in cellular automata theory. Glider Gun arises more frequently than Bbar8 and $\mathrm{H}$ gliders; nevertheless the quick interaction with other structures or chaotic regions avoids to form and conserve the glider because in a similar way with $\mathrm{H}$ glider, its period is very large.

\section{Concluding Remarks}

The list of gliders without extensions proposed by Cook is fully reproduced by means of collisions, the initial configurations used for obtaining this result have been constructed through the phases of each glider. In several paragraphs we have described the similarities between Rule 110 and The Game of Life, something interesting is that although Rule 110 has a one-dimensional evolution and its evolution rule is defined by eight neighborhoods, the global behavior is

\footnotetext{
${ }^{3}$ The glider speed is determined by the displacement of its cells between its period

${ }^{4}$ Soliton is a solitary wave with a nonlinear behavior which interacts with other waves conserving its form and speed, and suffering just small displacements in each collision 19
} 
very difficult to analyze. Another open question is to demonstrate that there are not more gliders in Rule 110.

The glider phases specify the horizontal measurement in periodic sequences aligned by ether, in fact they are sequences of the extended de Bruijn diagram [14], this diagram is useful to calculate all the periodic sequences of a given cellular automaton. In this way the de Bruijn diagrams may show the whole set of gliders in Rule 110, the problem is that these diagrams have an exponential growth for large gliders.

An interesting point of Rule 110 is the existence of complex behaviors in a periodic background, something that does not happen in The Game of Life which has a stable background. Rule 110 also defines several periodic backgrounds with other combinations of triangles. In these backgrounds the existence of others gliders can be discussed, although they must be carefully established because a small irregularity destroys these gliders and forms natural ether.

There is an unlimited number of interactions in Rule 110 and in the same way as The Game of Life, a time must pass to find new devices like blinkers, flip-flop configurations, structures eating other gliders, or large still-life regions. In this sense a complete and interesting study is presented in [5].

The analysis of automata with complex behaviors produced by the existence of gliders is an interesting area as Andrew Wuensche describes in 23. Wuensche defines a very practical process to filter gliders in periodic backgrounds of distinct cellular automata. A further work is to project Rule 110 in two and three dimensions for detecting other properties which have not been observed in one dimension. In the tridimensional case, the evolution space covered by tetrahedrons defining gliders and a periodic background must be spectacular.

Acknowledgements. In special to Matthew Cook, to Departamento de Aplicación de Microcomputadoras in UAP, the support of CONACyT with registry number 139509 and to Centro de Investigación Avanzada en Ingeniería Industrial in UAEH.

\section{References}

1. Andrew Adamatzky (Ed.), Collision-Based Computing, Springer, 2002 (ISBN 185233-540-8).

2. Elwyn R. Berlekamp, John H. Conway and Richard K. Guy, Winning Ways for your Mathematical Plays, Academic Press, 1982 (ISBN 0-12-091152-3) Vol. 2, chapter 25 .

3. Matthew Cook, "Universality in Elementary Cellular Automata," personal communication.

4. Matthew Cook, "Introduction to the activity of rule 110" (copyright 1994-1998 Matthew Cook), http://w3.datanet.hu/ ${ }^{2}$ cook/Workshop/CellAut/Elementary/Rule110/ 110pics.html, January 1999. 
5. Matthew Cook, "Still Life Theory," in New Constructions in Cellular Automata p. 93, (Santa Fe Institute Studies on the Sciences of Complexity) Oxford University Press, April 2003 (ISBN 0-1951-3717-5).

6. Martin Gardner, "Mathematical Games - The fantastic combinations of John H. Conway's new solitaire game Life," Scientific American 223, pp 120-123, 1970.

7. Jim Giles, "What kind of science is this?" Nature, 417 216-218 (16 May 2002).

8. Genaro Juárez Martínez, "Fases $\mathrm{f}_{i} i$ en la Regla 110," en preparación.

9. Genaro Juárez Martínez, "Solitones en el autómata celular unidimensional Regla 110," April 8, 2002

http://delta.cs.cinvestav.mx/ mointosh/oldweb/working.html.

10. Genaro Juárez Martínez and Harold V. McIntosh, "ATLAS: Collisions of gliders like phases of ether in rule 110,"

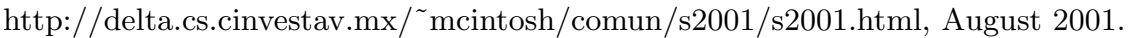

11. Genaro Juárez Martínez, Harold V. McIntosh and Juan Carlos Seck Tuoh Mora, "Estructuras periódicas cubriendo el espacio de evoluciones en el autómata celular unidimensional Regla 110,"

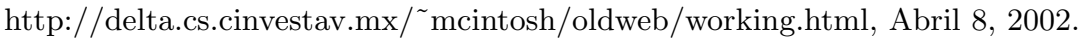

12. Genaro Juárez Martínez, Juan Carlos Seck Tuoh Mora and Harold V. McIntosh, "Reproducing the cyclic tag systems developed by Matthew Cook with Rule 110 using the phases $\mathrm{f}_{i \_} i$," in preparation.

13. Mariusz H. Jakubowski, Ken Steiglitz and Richard Squier, "Computing with Solitons: A Review and Prospectus," Multiple-Valued Logic, Special Issue on CollisionBased Computing, vol. 6, Numbers 5-6, 2001 (ISSN 1023-6627).

14. Harold V. McIntosh, "Linear cellular automata via de Bruijn diagrams,"

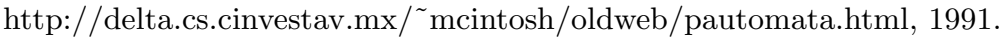

15. Harold V. McIntosh, "Rule 110 as it relates to the presence of gliders," http://delta.cs.cinvestav.mx/ mcintosh/oldweb/pautomata.html, January 1999.

16. Harold V. McIntosh, "A Concordance for Rule 110," http://delta.cs.cinvestav.mx/ ${ }^{\sim}$ mcintosh/oldweb/pautomata.html, April 2000.

17. Harold V. McIntosh, "Rule 110 Is Universal!,"

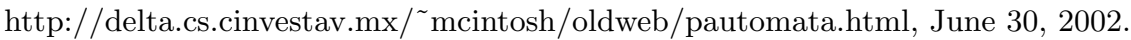

18. James K. Park, Kenneth Steiglitz and William P. Thurston, "Soliton-like behavior in automata," Physica D 19, 423-432, 1986

19. John Scott Rusell, "Report of Waves," Re. Brit. Assoc. for the Advancement of Science, pp. 311-390, 1844.

20. John von Neumann, Theory of Self-reproducing Automata, University of Illinois Press, Urbana and London 1966.

21. Stephen Wolfram, Theory and Applications of Cellular Automata, World Scientific Press, Singapore 1986.

22. Stephen Wolfram, A New Kind of Science, Wolfram Media, Inc., Champaign, Illinois, 2002 (ISBN 1-57955-008-8).

23. Andrew Wuensche, "Classifying Cellular Automata Automatically," Complexity, Vol. 4, no. 3, 47-66, 1999.

24. "OSXLCAU21" system is available for OpenStep and Mac OS X operating systems in http://delta.cs.cinvestav.mx/ ${ }^{\sim}$ mcintosh/comun/s2001/s2001.html, August 2001. 